\title{
Reduction of Cerebral Emboli: In vitro Study with a Novel Cerebral Embolic Protection Device
}

This article was published in the following Dove Press journal:

Medical Devices: Evidence and Research

\author{
Guy Haiman (iD) \\ Tamim Nazif ${ }^{2}$ \\ Jeffrey W Moses ${ }^{2}$ \\ Amit Ashkenazi ${ }^{1}$ \\ Pauliina Margolis (D) \\ Alexandra J Lansky $\mathbb{D}^{3,4}$ \\ 'Keystone Heart Ltd., Caesarea, Israel; \\ ${ }^{2}$ Department of Cardiology, Columbia \\ University Medical Center, New York, \\ NY, USA; ${ }^{3}$ Division of Cardiology, \\ Department of Internal Medicine, Yale \\ University School of Medicine, New \\ Haven, CT, USA; ${ }^{4}$ Department of \\ Cardiology, Yale Cardiovascular Research \\ Group, New Haven, CT, USA
}

\begin{abstract}
Aim: To assess the efficacy of the TriGUARD $3^{\mathrm{TM}}$, a novel cerebral embolic protection (CEP) device in reducing cerebral embolization by deflecting embolic debris away from the cerebral circulation using a quantitative in vitro model.

Methods and Results: This in vitro study assessed the ability of a cerebral embolic protection device to deflect embolic debris, by measuring the percent of particles and air bubbles, $200 \mu \mathrm{m}$ and $300 \mu \mathrm{m}$ in size, from entering the cerebral circulation compared to unprotected controls. A 3D printed silicone model of the ascending aorta, the aortic arch with its three major cerebral arteries and the descending aorta was connected to a custom-made simulator that mimics physiological pulsatile flow patterns of the left ventricle. Comparative analyses were used to assess the efficacy of the cerebral embolic protection device to deflect particles and air bubbles away from the major cerebral arteries. The percent of particles and air bubbles entering the major cerebral arteries was significantly lower with cerebral embolic protection compared to unprotected controls $(\mathrm{p}<0.0001)$. Cerebral protection resulted in 97.4-100\% reduction in air bubble counts, and $97.4-97.8 \%$ reduction in particle counts compared to unprotected controls.
\end{abstract}

Conclusion: This in vitro study used simulated physiologic flow conditions in an aortic arch model to demonstrate $>97 \%$ efficacy of the TriGUARD 3 CEP device, in reducing cerebral embolization of particulate and air bubbles of $200 \mu \mathrm{m}$ to $300 \mu \mathrm{m}$ in size.

Keywords: cerebral embolic protection device, CEP, deflection filter, in vitro study, transcatheter aortic valve replacement, TAVR, in vitro simulator

\section{Introduction}

Stroke occurs in up to $10 \%$ of patients after endovascular procedures and is one of the most feared and devastating complications due to its association with an extreme morbidity and mortality burden, which ranges from $25 \%$ to $30 \%{ }^{1-7}$ Clinically silent cerebral ischemia is more common after cardiac procedures occurring in virtually all patients after TransAortic Valve Replacement (TAVR) and has been associated with frailty, decline in physical function, cognitive decline, depression, and risk of subsequent stroke or TIA. ${ }^{8,9}$ Given the frequency and devastating implications of periprocedural stroke, methods to reduce cerebral embolism during cardiac interventions are sorely needed. During TAVR specifically, cerebral embolization of aortic plaque dislodged during retrograde instrumentation of the aortic valve is the primary etiology of periprocedural stroke. ${ }^{2}$ Several cerebral embolic protection (CEP) devices have been developed to reduce the risk of cerebral embolization. ${ }^{10-12}$ The Keystone Heart CEP Device is designed to protect all 3 major cerebral arteries and deflect embolic material away from the brain during TAVR to reduce the risk of ischemic brain injury.
Correspondence: Guy Haiman; AJ Lansky Email guy.haiman@keystoneheart.com; alexandra.lansky@yale.edu 
The aim of this in vitro study was to evaluate the deflection efficiency of the TriGUARD 3 device in an aortic arch model under simulated physiologic flow conditions.

\section{Methods}

\section{TriGUARD 3 Device}

The Keystone Heart TriGUARD 3 CEP device is designed to provide full coverage of all three major cerebral arteries during trans-catheter heart procedures. It is a temporary, sterile, single use, biocompatible deflection-filter, introduced transfemorally to the aortic arch, aimed to minimize the risk of cerebral injury by deflecting embolic debris away from the cerebral circulation during trans-catheter aortic valve replacement (TAVR) (Figure 1A and B). Once the device is in position, emboli and particulate matter are diverted away from the cerebral circulation. The device is designed to accommodate a variety of morphologies for stable deployment and provide complete cerebral coverage. The device is comprised of a nitinol frame and a dome shaped polymer mesh. The mesh has a nominal pore size of 115 $\mathrm{X} 145 \mu \mathrm{m}$, which allows adequate blood flow to the cerebral arteries while diverting emboli away from the cerebral circulation. Both the frame and the mesh are coated with hydrophilic heparin to reduce the risk of thrombogenicity and increase the lubricity of the deflection-filter.

\section{Experimental Setup}

To test the efficacy of TriGUARD 3 deflection, a customdesigned efficacy simulator (Figure 2A and B) consisting of a flow module that simulates physiological flow/pressure and an optical module designed was used to detect and quantify particles and air bubbles of variable sizes.

\section{Flow Module}

The flow module consists of a 3D silicone aortic arch model of a human aortic arch with its three cerebral arteries ((Innominate (IA), left carotid (LC) and subclavian (SC) arteries) was printed from Computed Tomographic Angiographic (CTA) images. The silicone model was connected to a pulsatile pump (Hemodynamics systems, IL) designed to maintain physiological flow of a $36 \% / 64 \%$ glycerol/deionized solution that mimics blood viscosity (3.5 mPa.s-1). A reservoir compliance chamber with a filter of $<10 \mu \mathrm{m}$ was incorporated into the module to prevent test particles from re-circulating through the system (only one run is allowed) or its connecting tubes (Tygon).

\section{Optical Module}

The optical module consists of two cuvette chambers; one for the cerebral arterial take-offs (Cerebral ascending) and the second for the descending aortic channel. The ascending and descending channels connect to a particle counting system comprised two high-speed cameras (IDS-UI $-3360 \mathrm{CP}-\mathrm{M}-\mathrm{GL}$ ). The high-speed cameras (3000 images per second) provided continuous recording of particles/air bubbles seeded in the flow loop as they pass through the cuvette chamber. Each image is processed, and the particles/air bubbles are counted and analyzed real-time by a custom-designed software. The software algorithm is written in Net 4.X using proprietary modules from C-Octopus to measure 200 (range:180-212) and 300 (range: $250-300) \mu \mathrm{m}$ particles and air bubbles and track their movement to avoid multiple counts. Air bubbles were inserted into the system solution by inducing a vortex in the reservoir tank. The image processing software was calibrated to count 200 and $300 \mu \mathrm{m}$ particles based on
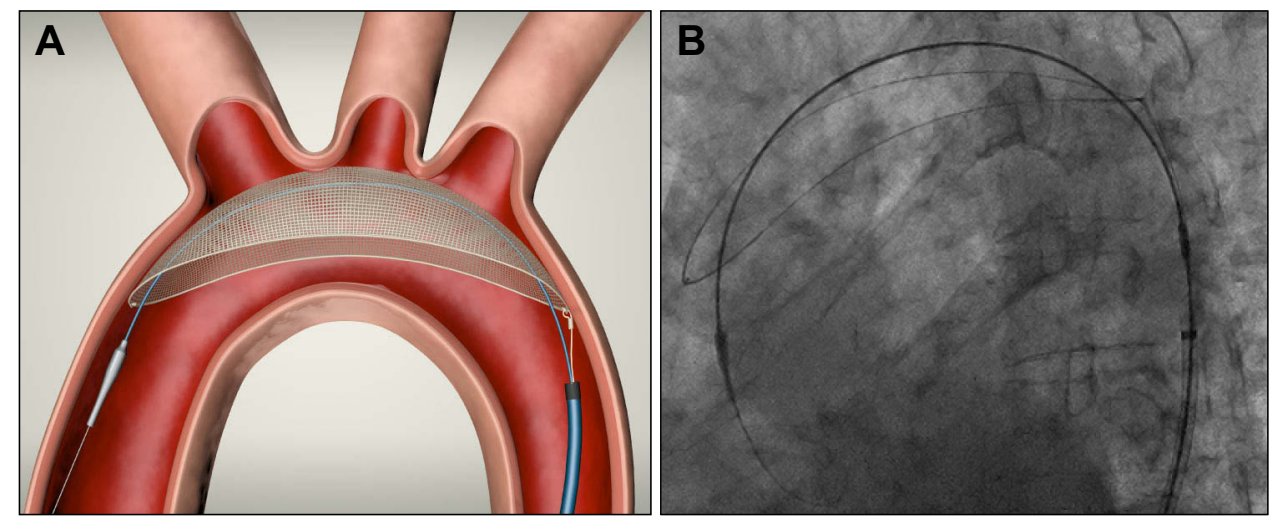

Figure I CEP device. (A) Positioned in the aortic arch providing full coverage of all three major aortic branches. (B) A fluoroscopic image of the device. 

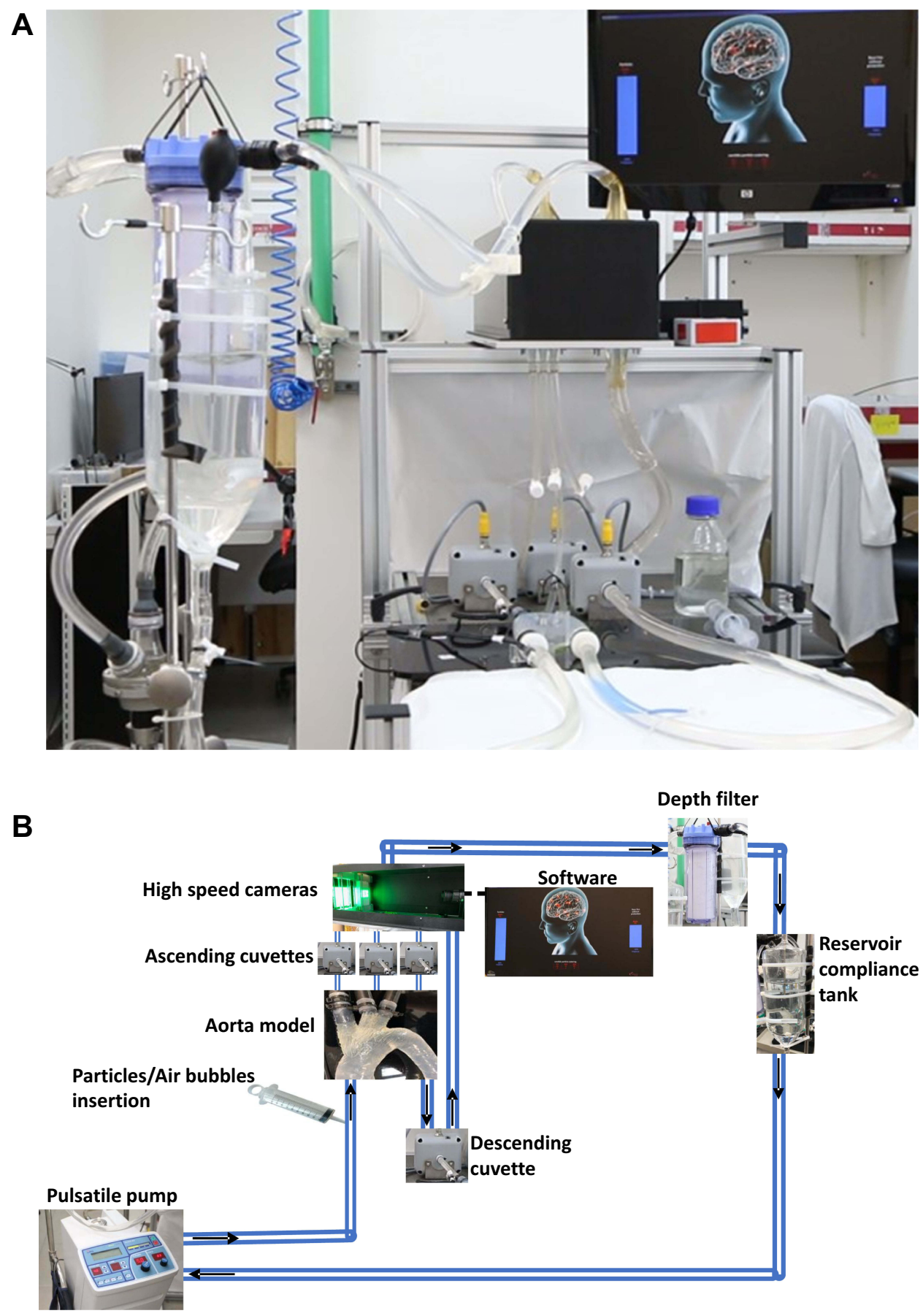

Figure 2 The in vitro efficacy simulator. (A) real and (B) Schematic presentation of the efficacy simulator. Black arrows indicate flow direction.

a prespecified pixel matrix of 5-8 pixels for 200 micron and 9-13 pixels for 300 microns detection.

The particle counting system was validated using image analysis software (Image-Pro Premier, Media Cybernetics, Japan), to compare the number of injected particles vs the counts detected in the ascending and descending channels, thus calculating the recovery rate of the counting system. Calculations demonstrated recovery rate of $91 \%$ to $95 \%$ for the $200 \mu \mathrm{m}$ and $93 \%$ to $97 \%$ for the $300 \mu \mathrm{m}$ particles.

\section{Protocol}

The CEP device was inserted in the flow loop by means of its delivery system and deployed following manufacturer's instructions. The device was deployed $3 \mathrm{~cm}$ proximal to the Innominate artery ostium. Prior to each measurement, 
the system was run for 5 mins to ensure that the fluid in the system was clean and no particle count was noted prior to the injection of the test particles. To prevent particles from attaching to the inner part of the syringe, a 1:1 mix of distilled water and hydrophobic polyethylene microspheres (each $500 \mathrm{~mL}$ of solution mixed with $5 \mathrm{mg}$ of dyed polymer; Cospheric, Santa Barbara, CA, USA) was used to inject the embolized solution into the aortic arch. Particles and air bubbles with diameters of 200 (range: $180-212 \mu \mathrm{m}$ ) and 300 (range: $250-300 \mu \mathrm{m}) \mu \mathrm{m}$ were selected for testing. Particles and air bubbles that flowed up the major cerebral arteries were labeled as cerebral arteries (CA) while those flowing downstream were labeled as descending aorta (DA). The study protocol included 6 experiments for particles and 3 experiments for air bubbles. Each experiment was conducted on different size $(200$ and $300 \mu \mathrm{m})$ with and without the CEP device in place (control).

\section{Definitions and Calculations}

Comparative analyses with vs without protection device (controls) were performed separately for particles and air bubbles, each for different sizes ( 200 and $300 \mu \mathrm{m})$ as they entered the major cerebral arteries. All analyses were applied for each experiment separately and for pooled data of all experiments.

The efficacy $(\eta)$ of the CEP device was defined: $\eta=100 \times\left(1-\frac{\% \text { of particles in filtered artery with CEP device }}{\% \text { of particles in filtered artery without CEP device }}\right)$

P-value of $5 \%$ or less (2-tailed) was considered statistically significant. The data were analyzed using the SAS ${ }^{\circledR}$ version 9.4 (SAS Institute, Cary North Carolina).Chisquare was used to statistically assess the differences in proportions of particles and air bubbles entering the major cerebral arteries and the descending channels with and without CEP device in place.

\section{Results}

The count and percent of particles entering the cerebral arteries were significantly lower and significantly higher in the descending aorta with the CEP device compared to controls for both $200 \mu \mathrm{m}$ and $300 \mu \mathrm{m}$ sizes $(\mathrm{p}<0.0001)$ (Table 1). Similar results were demonstrated with CEP using air bubbles of $200 \mu \mathrm{m}$ and $300 \mu \mathrm{m}(\mathrm{p}<0.05)$ (Table 2).

The pooled analysis ( 6 experiments for particles and 3 experiments for air bubbles) of different sizes (200 and $300 \mu \mathrm{m}$ ) demonstrated significantly lower counts and rate of air bubbles and particles entering the cerebral arteries and significantly higher counts and rate of air bubbles and particles in the descending aorta with CEP compared to controls $(\mathrm{p}<0.0001)$ (Table 3). Device efficacy (n) for protection of the cerebral arteries was $97.4 \%$ for $200 \mu \mathrm{m}$ and $100 \%$ for $300 \mu \mathrm{m}$ air bubbles, and $97.8 \%$ for $200 \mu \mathrm{m}$ and $97.4 \%$ for $300 \mu \mathrm{m}$ particles (Table 4 ).

Table I The Count and Percent (\%) of Particles in the Ascending Major Aortic Arches and the Descending Aorta, in $200 \mu \mathrm{m}$ and 300 $\mu \mathrm{m}$, with vs Without the CEP Device as Observed in Six Independent Experiments

\begin{tabular}{|c|c|c|c|c|c|}
\hline \multicolumn{6}{|c|}{ Particles: $200-\mu \mathrm{m}$} \\
\hline \multirow[t]{2}{*}{ Experiments } & \multicolumn{2}{|c|}{ Without CEP Device Count (\%) } & \multicolumn{2}{|c|}{ With CEP Device Count (\%) } & \multirow[t]{2}{*}{ Chi-Square ( $P$ value) } \\
\hline & Ascending Branches & Descending Aorta & Ascending Branches & Descending Aorta & \\
\hline I & $44(25.14)$ & $|3|(74.86)$ & $0(0)$ & $264(100)$ & $73.77(\mathrm{P}<0.000 \mathrm{I})$ \\
\hline 2 & $75(26.4 I)$ & 209 (73.59) & $7(1.69)$ & $407(98.31)$ & $99.26(\mathrm{P}<0.000 \mathrm{I})$ \\
\hline 3 & III (22.47) & $383(77.53)$ & $3(0.96)$ & $310(99.04)$ & $73.08(\mathrm{P}<0.000 \mathrm{I})$ \\
\hline 4 & $58(23.87)$ & $185(76.13)$ & I $(0.22)$ & 451 (99.78) & II3.75 (P<0.000I) \\
\hline 5 & $109(29.62)$ & $259(70.38)$ & $4(1.17)$ & $337(98.83)$ & $106.9(P<0.0001)$ \\
\hline 6 & III (24.24) & $347(75.76)$ & $0(0)$ & $246(100)$ & $70.78(\mathrm{P}<0.000 \mathrm{I})$ \\
\hline \multicolumn{6}{|c|}{ Particles: $300-\mu \mathrm{m}$} \\
\hline 1 & $76(24.44)$ & $235(75.56)$ & $0(0)$ & $233(100)$ & $66.18(\mathrm{P}<0.0001)$ \\
\hline 2 & $81(21.37)$ & $298(78.63)$ & $6(3.49)$ & $166(96.51)$ & $28.45(\mathrm{P}<0.000 \mathrm{I})$ \\
\hline 3 & $60(21.74)$ & $216(78.26)$ & $5(1.17)$ & $421(98.83)$ & $84.30(\mathrm{P}<0.000 \mathrm{I})$ \\
\hline 4 & $57(19.45)$ & $236(80.55)$ & $0(0)$ & $203(100)$ & $44.6 \mathrm{I}(\mathrm{P}<0.000 \mathrm{I})$ \\
\hline 5 & $100(27.47)$ & $264(72.53)$ & $\mathrm{I}(0.5)$ & 201 (99.5) & $64.49(\mathrm{P}<0.000 \mathrm{I})$ \\
\hline 6 & $99(23.4)$ & $324(76.6)$ & $0(0)$ & $321(100)$ & $86.65(P<0.0001)$ \\
\hline
\end{tabular}


Table 2 The Count and Percent (\%) of Air Bubbles in the Ascending Major Aortic Arches and the Descending Aorta, in 200 $\mu \mathrm{m}$ and $300 \mu \mathrm{m}$, with vs Without the CEP Device as Observed in Three Independent Experiments

\begin{tabular}{|c|c|c|c|c|c|}
\hline \multicolumn{6}{|c|}{ AIR Bubbles: $200 \mu \mathrm{m}$} \\
\hline \multirow[t]{2}{*}{ Experiments } & \multicolumn{2}{|c|}{ Without CEP Device Count (\%) } & \multicolumn{2}{|c|}{ With CEP Device Count (\%) } & \multirow[t]{2}{*}{ Chi-Square ( $P$ value) } \\
\hline & Ascending Branches & Descending Aorta & Ascending Branches & Descending Aorta & \\
\hline I & $65(26.64)$ & $179(73.36)$ & $4(2.13)$ & $184(97.87)$ & $47.53(\mathrm{P}<0.000 \mathrm{I})$ \\
\hline 2 & $129(27.80)$ & $335(72.20)$ & II (I.70) & $636(98.30)$ & $167.15(P<0.0001)$ \\
\hline 3 & $202(14.04)$ & I 237 (85.96) & $15(0.35)$ & $4318(99.65)$ & $559.66(P<0.0001)$ \\
\hline \multicolumn{6}{|c|}{ Air Bubbles: $300 \mu \mathrm{m}$} \\
\hline I & $12(10.81)$ & $99(89.19)$ & $0(0)$ & $63(100)$ & $7.31 \quad(P<0.01)$ \\
\hline 2 & $6(2.06)$ & $285(97.94)$ & $0(0)$ & $162(100)$ & $3.38(P=0.045)$ \\
\hline 3 & $19(3.72)$ & $492(96.28)$ & $0(0)$ & $2606(100)$ & $97.49(\mathrm{P}<0.000 \mathrm{I})$ \\
\hline
\end{tabular}

Table 3 Pool Analysis of Count and Percent, for Particles and Air Bubbles in Ascending Branches and Descending Aorta, in $200 \mu \mathrm{m}$ and $300 \mu \mathrm{m}$, with vs Without the CEP Device

\begin{tabular}{|c|c|c|c|c|c|c|}
\hline \multirow[t]{2}{*}{ Type } & \multirow{2}{*}{$\begin{array}{l}\text { Size } \\
(\mu \mathrm{m})\end{array}$} & \multicolumn{2}{|c|}{ Without CEP Device Count (\%) } & \multicolumn{2}{|c|}{ With CEP Device Count (\%) } & \multirow{2}{*}{$\begin{array}{l}\text { P-value by Chi- } \\
\text { Square }\end{array}$} \\
\hline & & $\begin{array}{l}\text { Ascending } \\
\text { Branches }\end{array}$ & $\begin{array}{l}\text { Descending } \\
\text { Aorta }\end{array}$ & $\begin{array}{l}\text { Ascending } \\
\text { Branches }\end{array}$ & $\begin{array}{l}\text { Descending } \\
\text { Aorta }\end{array}$ & \\
\hline \multirow{2}{*}{$\begin{array}{l}\text { Air } \\
\text { Bubbles }\end{array}$} & 200 & $396(18.44)$ & $|75|(8 \mid .56)$ & $30(0.59)$ & $5138(99.41)$ & $(\mathrm{P}<0.000 \mathrm{I})$ \\
\hline & 300 & $37(4.05)$ & $876(95.95)$ & $0(0)$ & $2831(100)$ & $(P<0.000 I)$ \\
\hline \multirow[t]{2}{*}{ Particles } & 200 & $508(25.12)$ & $15 \mathrm{I} 4(74.88)$ & $15(0.74)$ & $2015(99.26)$ & $(P<0.000 I)$ \\
\hline & 300 & $473(23.11)$ & 1573 (76.89) & $12(0.77)$ & 1545 (99.23) & $(P<0.000 I)$ \\
\hline
\end{tabular}

Table 4 Confidence Intervals and Efficacy Rates for Air Bubbles and Particles of $200 \mu \mathrm{m}$ and $300 \mu \mathrm{m}$

\begin{tabular}{|l|l|l|l|l|}
\hline Type & $\begin{array}{l}\text { Size } \\
(\boldsymbol{\mu m})\end{array}$ & $\begin{array}{l}\text { Efficacy } \\
(\%)\end{array}$ & $\begin{array}{l}\text { Lower } \\
\text { Confidence } \\
\text { Interval Limit }\end{array}$ & $\begin{array}{l}\text { Upper } \\
\text { Confidence } \\
\text { Interval Limit }\end{array}$ \\
\hline \multirow{2}{*}{$\begin{array}{l}\text { Air } \\
\text { bubbles }\end{array}$} & 200 & 97.42 & 96.24 & 98.29 \\
\cline { 2 - 5 } & 300 & 100.00 & 97.38 & 100.00 \\
\hline \multirow{2}{*}{ Particles } & 200 & 97.78 & 96.28 & 98.77 \\
\cline { 2 - 5 } & 300 & 97.42 & 95.42 & 98.68 \\
\hline
\end{tabular}

In the absence of the CEP device, the distribution of particles measured in each major cerebral artery was 35-48\% in the IA, $31-32 \%$ in the LCC and $21-23 \%$ in the LSC (Table 5).

\section{Discussion}

This in vitro study demonstrates that in a silicone aortic model with the CEP device positioned across the major cerebral arteries has $>97 \%$ efficiency in deflecting both particulate debris and air bubbles ranging from 200 to $300 \mu \mathrm{m}$ in size
Table 5 Total Amount and Distribution (\%) of Particles, in Different Sizes $(200 \mu \mathrm{m}$ and $300 \mu \mathrm{m})$, to Enter Each of the Ascending Major Aortic Arches

\begin{tabular}{|l|l|l|l|l|}
\hline \multirow{2}{*}{ Type } & \multirow{2}{*}{$\begin{array}{l}\text { Size } \\
(\boldsymbol{\mu m})\end{array}$} & \multicolumn{3}{|l|}{ Ascending Major Aortic Arches } \\
\cline { 3 - 5 } & & $\begin{array}{l}\text { IA Total } \\
(\%)\end{array}$ & $\begin{array}{l}\text { LCC } \\
\text { Total (\%) }\end{array}$ & $\begin{array}{l}\text { LSC } \\
\text { Total (\%) }\end{array}$ \\
\hline $\begin{array}{l}\text { Particles (6 } \\
\text { experiments) }\end{array}$ & 200 & $243(48 \%)$ & $158(31 \%)$ & $107(21 \%)$ \\
\cline { 2 - 5 } & 300 & $213(35 \%)$ & $153(32 \%)$ & $107(23 \%)$ \\
\hline
\end{tabular}

Abbreviations: IA, innominate artery; LCC, left common carotid; LSC, left subclavian.

compared to unprotected controls. Without CEP, $35-48 \%$ of particles entered the innominate artery, $31-32 \%$ entered the left common carotid artery, and $21-23 \%$ of the particles entered the left subclavian. These results support the advantage of full cerebral protection of the TriGUARD 3 rather than partial protection such as the Claret CEP (Medical/Boston Scientific) which protects only the brachiocephalic and left common carotid arteries, leaving the left subclavian artery unprotected. $^{13,14}$ This distinction is of clinical relevance, as the left subclavian artery is the major supply to the posterior 
portion of the Circle of Willis ${ }^{15,16}$ and therefore, cerebral infarcts may also be weighted towards the posterior circulation. $^{17}$

The design of TriGUARD 3 is aimed to provide stability, ease of delivery and a greater protective footprint to improve its safety, performance and efficacy. The TriGUARD 3 device is currently under clinical investigation in the REFLECT US IDE trial (NCT02536196), a randomized controlled, multicenter trial of patients undergoing TAVR with and without cerebral embolic protection.

\section{Study Limitations}

This in vitro study using a silicon aortic arch model under simulated physiologic flow, assessed the CEP device in an ideal fixed position across the cerebral vessels. It does not assess the potential interaction in a human with movement, the TAVR procedure delivery system, or variations in aortic anatomy.

\section{Impact on Daily Practice}

This in vitro study demonstrates approximately $97 \%$ efficacy of the CEP device in preventing particles and air bubbles entering the cerebral circulation. We demonstrated that about $1 / 5$ particles released in the ascending aorta will enter the left subclavian cerebral branch. This study provides an excellent in vitro setting to study the efficacy of any CEPD devices.

\section{Disclosure}

Tamim Nazif reports personal fees, equity from Keystone Heart and Venus MedTech, personal fees from Boston Scientific, outside the submitted work. Alexandra J Lansky reports grants from Yale School of Medicine, during the conduct of the study; Institutional Research support and travel fees from Venous Medical, outside the submitted work. The authors report no other conflicts of interest in this work.

\section{References}

1. McKhann GM, Grega MA, Borowicz LM Jr, Baumgartner WA, Selnes OA. Stroke and encephalopathy after cardiac surgery: an update. Stroke. 2006;37:562-571. doi:10.1161/01.STR.0000199032. $78782.6 \mathrm{c}$

2. Nuis RJ, Piazza N, Van Mieghem NM, et al. In-hospital complications after transcatheter aortic valve implantation revisited according to the valve academic research consortium definitions. Catheter Cardiovasc Interv. 2011;78:457-467. doi:10.1002/ccd.23018
3. Seeger J, Kapadia SR, Kodali S, et al. Rate of peri-procedural stroke observed with cerebral embolic protection during transcatheter aortic valve replacement: a patient-level propensity-matched analysis. Eur Heart J. 2019;40(17):1334-1340. doi:10.1093/eurheartj/ehy847

4. Selim M. Perioperative stroke. N Engl J Med. 2007;356:706-713. doi:10.1056/NEJMra062668

5. Fuchs S, Stabile E, Kinnaird TD, et al. Stroke complicating percutaneous coronary interventions: incidence, predictors, and prognostic implications. Circulation. 2002;106:86-91. doi:10.1161/01.CIR.000 0020678.16325.E0

6. Stortecky S, Windecker S. Stroke: an infrequent but devastating complication in cardiovascular interventions. Circulation. 2012;126:2921-2924. doi:10.1161/CIRCULATIONAHA.112.149492

7. Lansky AJ, Brown D, Pena C, et al. Neurologic complications of unprotected transcatheter aortic valve implantation (from the Neuro-TAVI Trial). Am J Cardiol. 2016;118:1519-1526. doi:10.10 16/j.amjcard.2016.08.013

8. Segal AZ, Abernethy WB, Palacios IF, BeLue R, Rordorf G. Stroke as a complication of cardiac catheterization: risk factors and clinical features. Neurology. 2001;56:975-977. doi:10.1212/WNL.56.7.975

9. Omran H, Schmidt H, Hackenbroch M, et al. Silent and apparent cerebral embolism after retrograde catheterization of the aortic valve in valvular stenosis: a prospective, randomized study. Lancet. 2003;361:1241-1246. doi:10.1016/S0140-6736(03)12978-9

10. Rodes-cabau J, Dumont E, Boone RH, et al. Cerebral embolism following transcatheter aortic valve implantation: comparison of transfemoral and transapical approaches. $J$ Am Coll Cardiol. 2011;57:18-28. doi:10.1016/j.jacc.2010.07.036

11. Lansky AJ, Schofer J, Tchetche D, et al..A prospective randomized evaluation of the TriGuard ${ }^{\mathrm{TM}}$ HDH embolic DEFLECTion device during transcatheter aortic valve implantation: results from the DEFLECT III trial. Eur Heart J.2015;36:2070-2078. doi:10.1093/ eurheartj/ehv191

12. Naber CK, Ghanem A, Abizaid AA, et al. First-in-man use of a novel embolic protection device for patients undergoing transcatheter aortic valve implantation. EuroIntervention. 2012;8:43-50.

13. Van Mieghem NM, El Faquir N, Rahhab Z, et al. Incidence and predictors of debris embolizing to the brain during transcatheter aortic valve implantation. JACC Cardiovasc Interv. 2015;8:718-724. doi:10.1016/j.jcin.2015.01.020

14. Van Mieghem NM, Van Gils L, Ahmad H, et al. Filter-based cerebral embolic protection with transcatheter aortic valve implantation: the randomized MISTRAL-C trial. EuroIntervention. 2016;12:499-507. doi:10.4244/EIJV12I4A84

15. Haussig S, Mangner N, Dwyer MG, et al. Effect of a cerebral protection device on brain lesions following transcatheter aortic valve implantation in patients with severe aortic stenosis. The CLEAN-TAVI randomized clinical trial. JAMA. 2016;316:592-601. doi:10.1001/jama.2016.10302

16. Baumbach A, Mullen M, Brickman AM, et al. Safety and performance of a novel embolic deflection device in patients undergoing transcatheter aortic valve replacement: results from the DEFLECT I study. EuroIntervention. 2015;11(1):75-84. doi:10.4244/EIJY15 M04_01

17. Fanning JP, Wesley AJ, Walters DL, et al. Topographical distribution of perioperative cerebral infarction associated with transcatheter aortic valve implantation. Am Heart J. 2018;197:113-123. doi:10.1016/j. ahj.2017.12.008 


\section{Publish your work in this journal}

Medical Devices: Evidence and Research is an international, peerreviewed, open access journal that focuses on the evidence, technology, research, and expert opinion supporting the use and application of medical devices in the diagnosis, monitoring, treatment and management of clinical conditions and physiological processes. The identification of novel devices and optimal use of existing devices which will lead to improved clinical outcomes and more effective patient management and safety is a key feature of the journal. The manuscript management system is completely online and includes a very quick and fair peer-review system. Visit http:// www.dovepress.com/testimonials.php to read real quotes from published authors. 